\title{
Highly transparent and antimicrobial PVA based bionanocomposites reinforced by ginger nanofiber
}

\begin{abstract}
Good transparency, antimicrobial, physical, and tensile properties of the biodegradable film can be necessary for food packaging. The aim of this study is to characterize these properties of the poly(vinyl alcohol) (PVA)/ginger nanofiber (GF) bionanocomposite film. This nanofiber of 0.21, 0.31 and $0.41 \mathrm{~g}$ in suspensions, was mixed with PVA gel using ultrasonication. After addition of ginger nanofibers, the bionanocomposite film shows antibacterial activity but does not have antifungi activity. Increasing the nanofiber into PVA increases significantly in tensile properties, water vapour impermeability, and moisture resistance. Tensile strength, the temperature at maximum film decomposition, and moisture resistance (after $8 \mathrm{~h}$ ) of the $0.41 \mathrm{~g}$ ginger nanofiber reinforced film were $44.2 \mathrm{MPa}$ (increased by $65.6 \%$ ), $349.4^{\circ} \mathrm{C}$ (increased by $7 \%$ ), and $6.1 \%$ (decreased by $18.7 \%$ ), respectively compared to pure PVA. With this nanofiber loading, the transparency of the bionanocomposite film decreased slightly. These results suggest this bionanocomposite film has potential in food packaging in industrial applications.
\end{abstract}

Keyword: Ginger nanofiber; Antimicrobial; Bionanocomposites; PVA film; Ultrasonication 